\title{
Maryam Borghée, Voile intégral en France. Sociologie d'un paradoxe
}

Paris, Éditions Michalon, 2012, 256 p.

Préface de Michel Wieviorka.Maryam Borghée, Voile intégral en France. Sociologie d'un paradoxe, Paris, Éditions Michalon, 2012, 256 p., Préface de Michel Wieviorka

Joëlle-Andrée Deniot

\section{OpenEdition}

Journals

Édition électronique

URL : http://journals.openedition.org/assr/27312

DOI : $10.4000 /$ assr. 27312

ISSN : $1777-5825$

Éditeur

Éditions de l'EHESS

Édition imprimée

Date de publication : 1 octobre 2015

Pagination : 267

ISBN : 978-2-7132-2515-4

ISSN : 0335-5985

Référence électronique

Joëlle-Andrée Deniot, « Maryam Borghée, Voile intégral en France. Sociologie d'un paradoxe », Archives de sciences sociales des religions [En ligne], 172 | octobre-décembre, mis en ligne le 17 mai 2016, consulté le 24 septembre 2020. URL : http://journals.openedition.org/assr/27312 ; DOI : https:// doi.org/10.4000/assr.27312

Ce document a été généré automatiquement le 24 septembre 2020.

() Archives de sciences sociales des religions 


\section{Maryam Borghée, Voile intégral en France. Sociologie d'un paradoxe}

Paris, Éditions Michalon, 2012, 256 p.

Préface de Michel Wieviorka.Maryam Borghée, Voile intégral en France. Sociologie d'un paradoxe, Paris, Éditions Michalon, 2012, 256 p., Préface de Michel Wieviorka

Joëlle-Andrée Deniot

\section{Résonances}

2 «Le balancier des hanches / La poitrine qui penche / Flou souvenir des pleins des creux / Les doigts longs les mains blanches / Disparus sous les manches / Rideau tiré sur les cheveux / La bouche qu'on enterre / Qui ne doit que se taire / Le monde interdit pour les yeux / Sur ce corps qui s'efface / J'ai regardé en face / L'hypocrisie offerte à Dieu / J'ai attendu, attendu / Et je l'ai aperçue / La femme cachée sous le tissu [...]» chante avec délicatesse Jeanne Cherhal, jeune artiste de la scène française. Composée et enregistrée en 2008, la chanson mélodieuse et à l'écriture raffinée milite en nuances en faveur d'une libération de «l'habit-linceul» sur laquelle se clôt le dernier couplet. Les chansons sont comme l'on sait des sources utiles renseignant sur

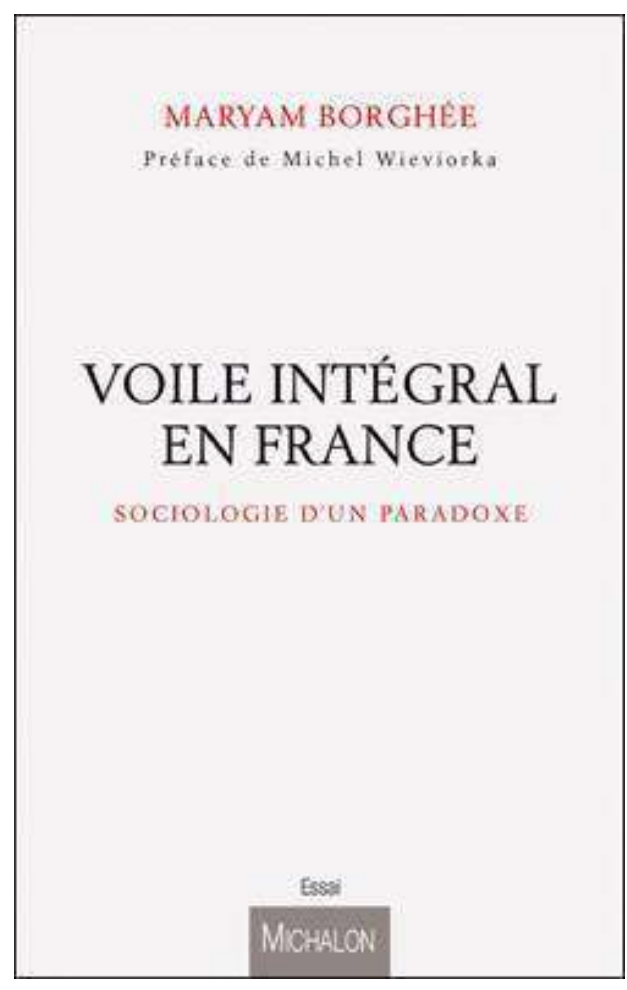
l'esprit du temps et cette chanson douce, loin des caricatures, des slogans pontifiants, nous dit l'unique voie du progrès, l'impossible imaginaire d'une autre liberté... 
3 «[...] Et soudain elle prit / La main de son mari / Il s'était passé quelque chose / Elle arracha le voile / Jeta la longue toile / Et je vis sa métamorphose / Les cheveux libérés / Les genoux desserrés / Elle était redevenue femme [...]».

4 Cette mélodie n'est pas inscrite dans l'étude de l'auteur mais elle lui fait écho car elle introduit en pointillé à des questions centrales :

5 - Pourquoi le débat en France s'est-il focalisé avec autant de force, aussi bien du côté d'un féminisme de bon aloi que du côté d'un occidentalisme sans recul sur ce registre de la parure des femmes?

6 - Comment ce voile peut-il apparaître comme voile de la honte quand celles qui le portent lui donnent une valeur non seulement esthétique mais surtout axiologique?

7 - N'est-il pas étrange que nous ne parvenions pas à soumettre au doute (clef de voûte de notre rationalité) le refus de notre inconscient collectif à penser des formes de pudeur, de rapport à la sexuation, de rapport à la sublimation de soi qui s'écartent radicalement de nos attentes?

8 C'est dans le cadre du colloque organisé par le Lestamp, en juin 2012, sur des Hommes, des Femmes, Inerties et métamorphoses anthropologiques que nous découvrons les travaux de Maryam Borghée venue à cette occasion nous exposer les principaux thèmes de sa réflexion sociologique à propos de ces musulmanes qui, en France, décident d'arborer - de façon intermittente ou constante - dans l'espace public, le voile qui fait scandale, à savoir le voile masquant non seulement le corps, les mains mais surtout le visage, ce lieu par excellence de la révélation du Sujet qui fait face, de l'intersubjectivité communément partagée. Femmes noires, femmes ombres, femmes tissus... provoquant « nos valeurs d'égalité », «notre modernité » et «notre postmodernité » tout autant! Et pourquoi pas la raison même, à moins que ce ne soit notre ethnocentrisme ou notre aveuglement à toute réinvention du religieux. À plus d'un titre, celui de l'interpellation $d u$ féminin, du féminisme, celui des rapports sociaux entre hommes et femmes, celui géopolitique d'un mimétisme mondialisé et antagoniste des croyances, la recherche de Maryam Borghée croisait la voie critique de ce colloque qui osait parler d'une humanité sexuée plutôt que d'une post-humanité genrée !

Ce livre, dans le contexte du pesant conformisme régnant, est d'abord un acte courageux dont l'objet se présente à première vue comme presque inaccessible, tant il est saturé d'a priori aux multiples sources : ceux issus de l'emballement étatique, des débats parlementaires du moment (Loi de 2011 interdisant dans l'espace public toute "tenue destinée à dissimuler le visage »), ceux issus de la dramatisation médiatique, ceux issus des différents mouvements et personnalités se voulant toujours porte-parole des Lumières, ceux issus d'une crainte diffuse de l'Islamisme, ceux issus du rejet sans appel et non sans cause de cette oppression radicale symbolisée par la burqa, emblème du régime des talibans.

10 Il fallait donc à l'auteur souligner les confusions et tenter de les démêler. Méconnaissance des cultures du monde musulman, de leur étendue, des liens tissés entre culture et religion, ignorance des fondements canoniques et de la jurisprudence islamique en matière de voilement du corps féminin alimentent cette focalisation des observateurs pressés sur le seul signifiant burqa. Sans entrer dans des débats théologiques, l'auteur commence par guider le lecteur dans cet inconnu, elle insiste sur la clarification des termes employés, elle montre comment sur un usage démagogique des mots se greffent des glissements sémantiques qui amplifient erreur et rumeur. 
11 Mais il fallait également imaginer - et là se trouvait le plus complexe - qu'il y avait quelque chose d'un sens existentiel singulier à découvrir qui se situait hors de portée des idéologies convenues de la soumission, de l'aliénation ou de tout autre concept visant à nommer la dépossession de soi : seule interprétation autorisée à appréhender le comportement de ces femmes apparemment sans visage.

12 L'auteur s'appuie sur une enquête ethnographique qui débute en 2008 et qui - sous divers angles et manières - donne la parole aux intéressées : suivi d'échanges sur les réseaux sociaux de sites communautaires, questionnements par courriel, observation directe de mosquées salafistes à Montreuil, à Vitry-sur-Seine, aux Mureaux, à Nanterre, etc., collecte de nombreux entretiens informels et de 24 entretiens de type semidirectifs. Au bilan de cette enquête, on peut enregistrer la rencontre, à niveaux variés, d'une soixantaine de femmes portant occasionnellement ou quotidiennement le niqâb (voile facial d'inspiration saoudienne laissant seulement les yeux à découvert).

13 Deux axes d'approche empirique se dégagent, qui convergent vers deux formes d'intelligibilité complémentaire. La première est synthétique ; elle découpe des idéauxtypes à partir d'une sociographie de ces jeunes femmes réislamisées ou converties. De milieux sociaux hétérogènes, les unes et les autres - assez peu soucieuses, voire entièrement détachées de l'arrière-plan politique salafiste de cette prescription vestimentaire - sont à la recherche d'une ligne de conduite valorisante se référant de préférence à l'image d'un islam universel. Effort d'apprentissage de la doctrine, quête d'une plus grande rigueur morale ou bénéfice espéré d'un beau mariage dessinent pratiques, implications et figures différenciées de ces croyantes ainsi que des modes différenciés de ruptures par rapport à l'environnement familial et sociétal. Mais lettrées, dévotes ou futures épouses (les trois termes n'étant d'ailleurs pas exclusifs), elles visent toutes un anoblissement de leur statut, une sortie de crise "par le haut" symboliquement et socialement. Car si situation critique il y a, elle précède et accompagne fréquemment ce choix risqué, socialement extrême, du voile intégral, que l'une des interviewées, ancienne chrétienne, désigne par l'heureuse expression de " cloître »; l'auteur poursuit l'image et parle alors de « cloître ambulant » (p. 90-91).

Et nous entrons là dans le développement sans doute le plus radicalement neuf et le plus émouvant aussi, à savoir l'analyse des récits biographiques livrés par les interlocutrices de la sociologue. Des blessures. Des violences. Des révoltes. Des parcours globalement rudes, des vies d'enfants, d'adolescentes malmenées. Pourtant le plus décisif ne se situe pas dans ces déchirures dont les témoignages trouveraient des homologies dans bien d'autres situations socioculturelles, mais précisément dans cette mobilisation spécifique d'imagination et de raison de chacune en vue d'une métamorphose de leur être. Le mot n'est pas trop fort, car il s'agit souvent au sens littéral de renaître à soi d'abord, puis au monde. Tout ici s'inverse, nous les avions adjugées comme totalement hétéronomes et nous découvrons des femmes rebelles placées dans un trajet de réappropriation de leur personne. Le passage de l'assujetti au sujet ne va jamais de soi. Quand la conquête se heurte à de nombreux obstacles, comme dans leur cas - « la jeune femme est méconnaissable, elle devient étrange et étrangère à sa famille» (p. 155) - le Sujet doit pour affronter l'adversité s'héroïser et c'est en sujets héroïques que nous les entendons parler de leur combat : «Les sœurs qui le portent [le niqâb] en France, je les soutiens, elles ont vraiment la foi, pour supporter les critiques, les regards [...] Ce sont des pionnières ! Elles se sacrifient ! Elles font tout le travail pour que la société finisse par accepter »(p.196). 
15 Toutefois l'auteur ne se contente pas de retraduire ce matériau déjà très riche des entretiens et des observations - le livre s'ouvre sur une description fort bienvenue d'une " journée avec ces femmes en noir » mettant en relief les climats de convivialité, les ambiances d'échanges sororaux noués dans ce cadre - elle apporte aussi une herméneutique tout à fait intéressante. En effet loin de se restreindre aux seuls auteurs classiques et contemporains de la discipline sociologique pouvant entrer en dialogue avec les thèmes de la religion, de l'individuation, du corps, de la pudeur, du stigmate, l'auteur s'aventure vers d'autres schèmes interprétatifs issus de l'anthropologie du sacré, de la philosophie, et cela essentiellement pour les questions de la volonté avec Paul Ricœur, du visage avec Emmanuel Levinas, du sublime avec Emmanuel Kant.

Car tissu salvateur, tissu matriciel - les femmes interrogées parlent de "cocon », de «bulle »- d'où le sujet va pouvoir se refonder, ce voile et cet acte de séparation qu'il marque captent une force de significations inouïes dont les récits les plus révélateurs et les plus enchantés sont ceux de la toute première rencontre : «Elle faisait comme de la lumière sur le trottoir, y'avait un peu de vent, le tissu il volait, elle était comme... illuminée. Elle était avec elle-même, dans son esprit. Je ne voyais qu'elle, elle rayonnait... Bon, c'était dans le XVIII ${ }^{\mathrm{e}}$, c'est pas les Champs-Élysées, mais on dirait qu'elle avait ramené un arc-en-ciel » (p. 199). Une telle manière de nommer l'épiphanie de la grâce entre choc amoureux et élan mystique ne laisse pas indifférent, elle indique la profondeur du sens de ce langage d'une enveloppe corporelle investie à la fois comme parure et comme auréole. On peut regretter dans la foulée de ces remarques pertinentes sur ce tissu-texte de la renaissance, l'absence de références aux travaux de Didier Anzieu sur le Moi-peau.

17 Au-delà de l'objet d'étude, cette recherche à portée sociétale aigüe nous conduit vers une réflexion plus universelle sur les difficultés politiques liées au traitement des cultures de l'intime ainsi qu'au contresens et à l'impasse où conduit leur déni. Au centre la question du visage, ce foyer ontologique de l'identité, de l'authenticité dans nos montages culturels. Qu'est-que le visage? Levinas est le seul philosophe (tout autant influencé par "l'intrigue du religieux » que par la phénoménologie) qui se soit attaché à penser cette donnée sensible et transcendante de notre apparence, où s'ancre via le regard qui vous fixe en sa fragilité irrévocable, "sa pauvreté essentielle ", une éthique de l'Altérité et de la responsabilité. Mais comme le note avec justesse Maryam Borghée, ce concept du visage ne renvoie pas à ses traits, à sa morphologie, à sa plastique, mais à une idéalité, à l'infini de la dette et du don. Aussi comme le souligne également l'auteur, Levinas ne saurait servir de parade théorique et moralisante pour justifier l'interdiction du voile intégral! Reste que la réflexion lévinassienne s'éloignant en cela du dogme judaïque - intègre philosophiquement dans son éthique le thème de l'Incarnation chrétienne, de la proximité de Dieu dans le visage de mon prochain. Chaque face porte bien l'écho de la Sainte Face, nous sommes dans une logique de l'épiphanie, de ce qui se révèle et apparaît pour être autrement; cet angle aurait pu introduire quelques contradictions que l'auteur évite. Ces interlocutrices voilées ne sont bien sûr pas sans visage par ce simple retrait visuel; leur voix, leur geste, leur démarche, devenant d'ailleurs miroirs des traits provisoirement masqués. Toutefois une réelle ambiguïté subsiste, qui aurait sans doute mérité plus d'attention encore.

18 Il ne faut pas se méprendre, cet essai ne cède pas au plaidoyer pour le voile intégral car il est loin de cacher toutes les contradictions inhérentes à ce port du voile consenti. 
"Dans des démocraties séculières des femmes ressentent le besoin de cacher leur visage aux yeux du monde [...] embrassent l'islam et adoptent délibérément le voile intégral. Toutefois "délibéré" ne signifie pas "libre" ou "libérée": leur pratique survient dans un contexte de vulnérabilité [...]. Toute leur ambivalence "est de vouloir s'accomplir en même temps que de se mettre en retrait" "(p.203). On pense immédiatement, dans le cas d'un maintien sur la longue durée d'une telle pratique, à leur mise hors champ du travail salarié, leur ôtant une grande part de l'autonomie qu'elles souhaitaient acquérir. Cependant la définition même de l'Autre n'est-il pas de garder tout un pan de son opacité.

Et cela n'en devient-il pas plus nécessaire encore au regard des événements les plus récents qui relancent - dans l'effroi certes justifié mais aussi dans la plus grande des confusions - « la guerre des civilisations » ? À la suite de la publication de son ouvrage, l'auteur rencontra quelques difficultés et des résistances bien prévisibles, sans pour autant réagir sur le vif. Dans le climat actuel, il est raisonnable de se demander si une telle étude ne se heurterait pas à des obstacles encore bien plus nombreux. D'audacieuse, cette recherche est peut-être devenue taboue. Sa lecture n'en est alors que plus pertinente. 